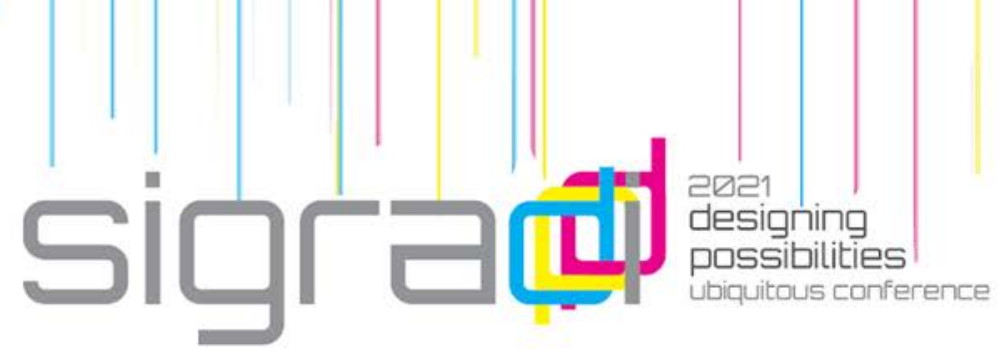

\title{
Deciphering and Forecasting Characteristics of Bodrum Houses Using Artificial Intelligence (AI) Approaches
}

\author{
Veli Mustafa Yönder, Fehmi Doğan, Hasan Burak Çavka \\ İzmir Institute of Technology, Turkey \\ veliyonder@iyte.edu.tr \\ fehmidogan@iyte.edu.tr \\ hasancavka@iyte.edu.tr
}

\begin{abstract}
Computer vision (CV), artificial intelligence (AI), machine learning (ML), and deep learning (DL) applications, which are among the rapidly emerging and growing technologies, have the potential to be effectively used in the fields of architecture and construction. These applications are used not only in the field of architectural design development and construction site tracking but also to analyze and predict the architectural properties of existing buildings and heritage classification. This paper aims to classify and analyze the façades of Bodrum houses by using deep learning models, comprehensive relational database (RDB), and artificial neural network based clustering methods. Through the use of the above-mentioned methods, we managed to cluster Bodrum houses' façade attributes in five groups and testing image classification models in three different classifiers.
\end{abstract}

Keywords: Image processing, Deep learning (DL), Classification, Hierarchical cluster analysis, Artificial neural networks (ANNs)

\section{Introduction}

Artificial intelligence provides a huge opportunity in the architectural constituent classification and clustering with providing a more informed approach. In this study, we are proposing to demonstrate how Al could be used in identifying the characteristics of Bodrum (Turkey) houses and in future studies in projecting potential avenues of evolution of these houses based on identified procedural changes in the vernacular.

The classification and clustering process of Bodrum houses are important to conserve the cityscape. The architectural features of Bodrum houses have idiosyncratic architectural attributes that need to comply with the municipal 
ordinances. In this study, it is aimed to classify houses based on façade photographs and cluster them with numerical façade features. The investigated Bodrum houses have common attributes, such as two-storey height and located in harmony with topography, white façades, and regulated and precise façade proportions. A database which contains numerical features of façades was created for clustering operations. With NN-based clustering operations, the database elements were divided into five separate groups. Moreover, SqueezeNET architecture and Inception-v3 architecture were used together with Support Vector Machine (SVM), Random Forest (RF), and Logistic Regression (LR) learners for image classification processes. The highest scores were 0.898 for AUC (Area Under Curve), 0.792 for classification accuracy (CA), 0.791 for F1, 0.804 for precision, and finally 0.792 for recall.

\section{Literature Review}

According to Gero (1996), it is normal that artificial intelligence and its methods are effective in the field of computer-aided design. Sophisticated neural network models are fundamental modules of the Al development stages. The existence of aggregated and scaled data is one of the factors that can trigger the beginning of digital transformation procedures and data-driven decision-making strategies. In this context, it is significant to understand existing data structures and establish meaningful relationships among them, forming predictive models and deciphering hidden patterns. The rules between the components in the raw dataset may not be clearly defined/programmed, accordingly artificial neural networks (ANNs) and learning methods that assist at this point aid us to make sense of the relationship between the parameters. Naturally, this procedure can be thought of as a "black-box or grey-box model", but these networks can identify "nonlinear relationships" between data in solving engineering problems and contribute to the specific resolution (Tayfur 2012, p.11). Contrastingly, neural networks have the potential to be used not only in solving engineering design issues, but also in computational design, digital fabrication and prototyping, computer-aided design (CAD), computer-aided manufacturing (CAM), architectural style classification, generating architectural artificial plans, producing artificial façades, façade segmentation, and building shape recognition domains. Artificial intelligence provides a huge opportunity in maintaining the vernacular heritage of traditional settlements and in providing a more informed approach to how these traditions could be transferred to future generations. Artificial intelligence techniques are used in different studies to recognize and classify the architectural style of buildings (Obeso et al. 2016; Yi et al. 2020). Moreover, computer vision techniques have great potential to classify architectural designs and designers' styles based on 
image similarities and differences. It is a noteworthy task to compare the architectural attributes of the buildings and forming novel types with expert references in architectural discourse. It is necessary to analyze the similaritydissimilarity of the geometric ratios of the building types and to make inferences according to the results of the analysis. The stages of classification and grouping of architectural elements have an active role in creating architectural typology. Wei et al. (2018) gathered building images from eighteen different cities via Google Street View and analyzed the architectural styles of residential buildings with computer vision techniques. In the study of $\mathrm{Yi}$ et al. (2020), they performed tests for image classification and style predictions of United States house in several architectural styles with the assistance of deep convolutional neural networks and a fully-connected neural network by utilizing Google images. In the study of Obeso et al. (2016, p.16), they experimented with a sophisticated convolutional neural network "to classify images of the architectural style of Mexican buildings in three different classes, pre-hispanic, colonial, and modern". Meltser et al. (2017) created a large dataset and they used the $\mathrm{CNN}$ model in feature extraction. Furthermore, K-nearest neighbor (KNN), enhanced fisher model KNN, and support vector machine (SVM) are the classifiers they use (Meltser et al. 2017). In style classification studies, the input data can be historical buildings as well as modern buildings and their designers. Huang et al. (2020) prepared a dataset of façade photographs of the buildings of five famous architects from the internet. This dataset, consisting of hundred images, has been studied for designers' classification processes through segmentation phases and pre-trained-CNN models such as SqueezeNET, Inception-v3, VGG-16, etc. (Huang et al. 2020). It is also possible to perform style classification, forecasting, and clustering analysis by designing numerical databases. In the study of Xia et al. (2020), classification and style forecasting operations were carried out through survey based quantitative data about houses.

\subsection{Key Frameworks of AI}

Artificial neural networks, one of the fundamental concepts of artificial intelligence, can imitate some of the cognitive actions of the nervous system through intelligent ways in a unique system. An artificial neuron is made up of "input vector, synaptic weights, bias, output, net information, and activation function" (Tayfur 2012, p.13). It is clear that they are very effective in carrying out learning actions and accomplishing novel results in various challenging areas. Their architecture consists of the input layer, output layer, hidden layer, and bias (Huang 2003). Machine learning, which is a sub-branch of the artificial intelligence family, is older than deep learning in terms of implementation areas and origin. It includes the methods of processing data with supervised, unsupervised, hybrid, and reinforcement learning algorithms (Fyfe 2000). According to computer scientist Tom Mitchell (1997, p.11), machine learning is critical for "data mining, difficult-to-program applications, 
and customized software applications". In classic software development, requests and tasks organized by the designer/developer are defined, while in ML processes, the software can learn from big data. In this context, working with cumulative data and making inferences about solving a well-defined problem can be achieved through these algorithms.

Another term is deep learning, a sub-branch of machine learning, consists of more hidden layers, hence it is called "deep", and it is different from shallow networks. In addition to basic statistics calculations and algebraic operations, mathematical expressions such as Tensors and gradient descent are part of the numerical side of deep learning. It is used in voluminous diverse fields and disciplines, from engineering to medicine, for computer vision applications and natural language processing. Convolutional neural networks (ConvNet), which are the intersection point of computer vision and deep networks, were also tested in this study. These networks, which are effective in semantic segmentation, image classification, scene recognition, predicting objects, etc., consist of many layers in terms of both number and diversity. Therefore, the design and arrangement of layers are determined by the nature of the problem and its possible elucidation approaches. One of the earliest developed networks, the seven-layer LeNET-5 (convolutional layers, subsampling layers, and fully connected layers) was created to recognize grayscale handwritten digits (LeCun et al. 1998). When constructing this type of network, input layer, "convolution, pooling or subsampling, activation function, connections and layers, and regulation" phases are considered (Elmas 2021, p.171). Images (color or grayscale), videos, and point cloud files can be submitted into input layers on these networks. The convolution layer, which is one of the principal components of the structure, is aimed to extract the attributes of the input image by applying filters. Activation functions such as rectified linear units (ReLu), Sigmoid, Swish, Softmax, Softsign, Softplus, Exponential linear units (ELUs), Maxout, ELisH, and Hyperbolic tangent (Tanh) are used for calculations between some layers in neural networks (Nwankpa et al., 2018).

Another important point to consider when creating a network is "connections" between input, output, and hidden layers, such as a fully connected layer. After the LeNET architecture was developed, many deep networks with more elegant structures and more layers were developed. AlexNet, an eight-layer deep neural network (convolutional layers and fully connected layers) designed for classification purposes, was developed by Krizhevsky et al. in 2012 and tested on complex colored images, eventually, the developers achieved successful results. Nineteen-layer model VGG (Simonyan and Zisserman, 2014), GoogLeNET with twenty-two-layer network structure (Szegedy et al., 2015), and one hundred fifty-two layers network ResNet (He et al., 2016) are examples of state of art networks built with the ConvNet structure for image classification purposes. Furthermore, Inceptionv3, developed by Szegedy et al. (2016), performs image recognition processes with ConvNet structure. SqueezeNET, another network trained on 


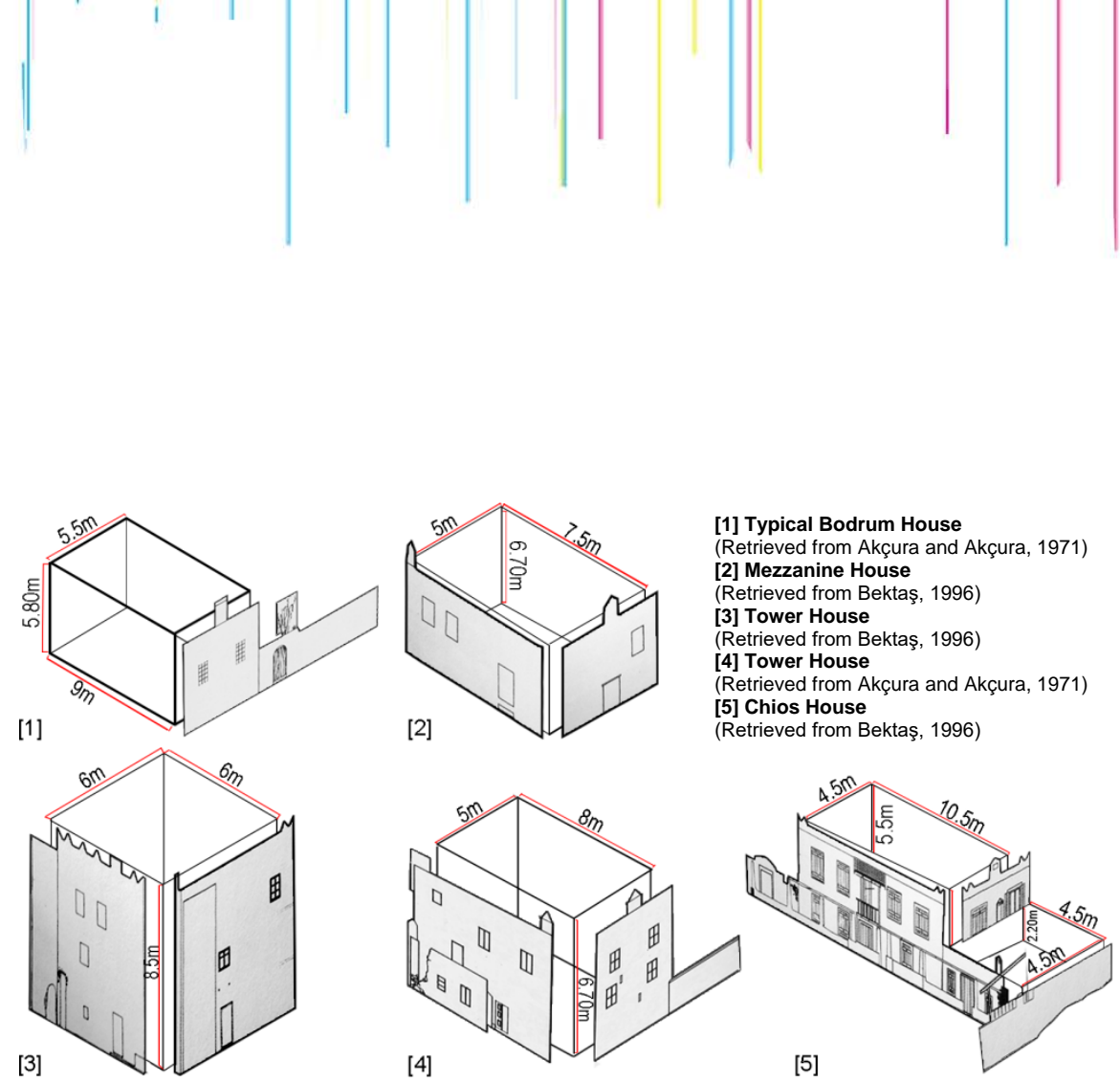

Figure 2. Conventional Bodrum houses

Source: Adapted from Bektaş (1996) and Akçura and Akçura (1971)

\section{Methodology}

The methodology of this study consists of two main stages (Figure 3). The first stage is about the neural network-based clustering analysis by utilizing the numerical properties of the façade elements of Bodrum houses. Synchronically, the second is about the work packages to classify the façade images of houses through machine learning algorithms and image embedding with deep neural network models. In this study, Intel Core(TM) i5-10300H CPU @ 2.50GHz 8GB of DDR4 RAM and Nvidia GTX 1650 4GB of GDDR5 were used as the hardware. Moreover, the digital tools employed in this study were GNU Image Manipulation Program (GIMP, 2019), PerspectivelmageCorrection (Sourceforge, 2021), Orange canvas with Python scripting and visual programming framework (Demšar et al., 2013), NeuroXL ${ }^{\circledR}$ environment (Olsoft, 2016), Xlstat $\AA$ (Addinsoft, 2019), Microsoft $\AA$ Office $2019^{\mathrm{TM}}$ and add-ins for database designing and analysis, and finally Daniel's XL Toolbox (Kraus, 2014), respectively. The data editing stage, which is the subsequent part after the data collection section, consists of cleaning, sorting, and arranging the various façade images obtained. After the editing, the analysis and numerical features of the façade elements were obtained, and database was designed. Since the database contains different data types such as areas and numbers, numeric values were aligned between $[0,1]$ via normalization operations. Normalization formulation (Source: Adapted from Wikipedia Contributors, 2019):

$\left\{X_{1}, X_{2}, X_{3}, X_{4}, X_{5},, X_{i}\right\} \in N$; they are members of input class.

$\left\{X_{n}\right\}=\left[\left(X_{i}-X_{\text {Min }}\right) \div\left(X_{\text {Max }}-X_{\text {Min }}\right)\right]$. Thus, " $X_{n}$ " $\in[0,1]$ 


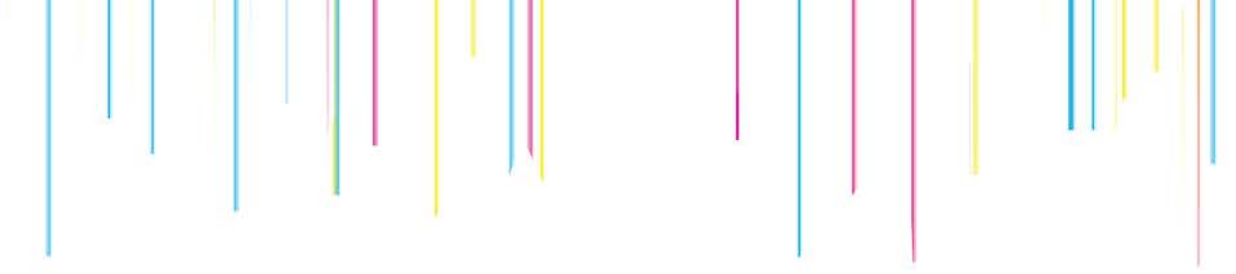

are very close to the average values (except the "terrace" and "façade material") (Figure 5). According to the classification results of the façade images, the highest classification accuracy value was when SqueezeNET and SVM were operated together, and the lowest value was the situation where SqueezeNET and RF were operated together (Figure 6). Furthermore, confusion matrix and Receiver operating characteristic (ROC) analysis results were calculated (Figure 6 and 7).

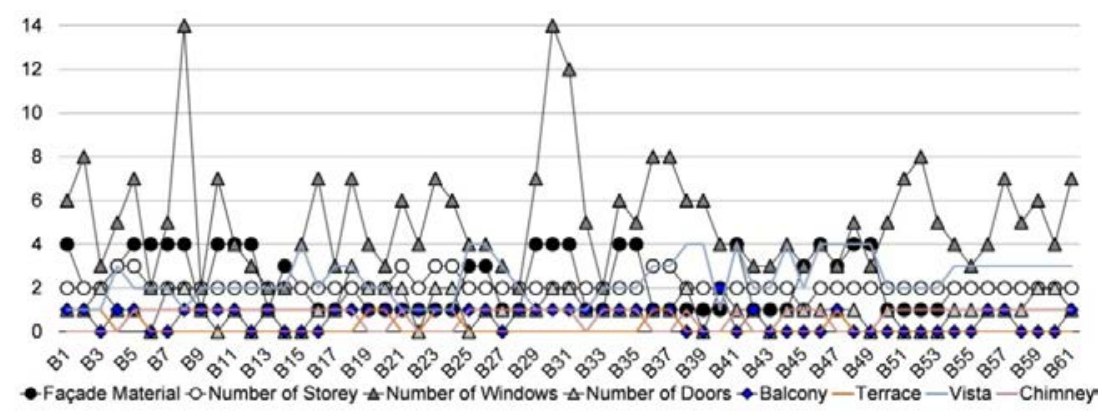

Figure 4. Data visualization of Bodrum houses façade elements Source: Authors

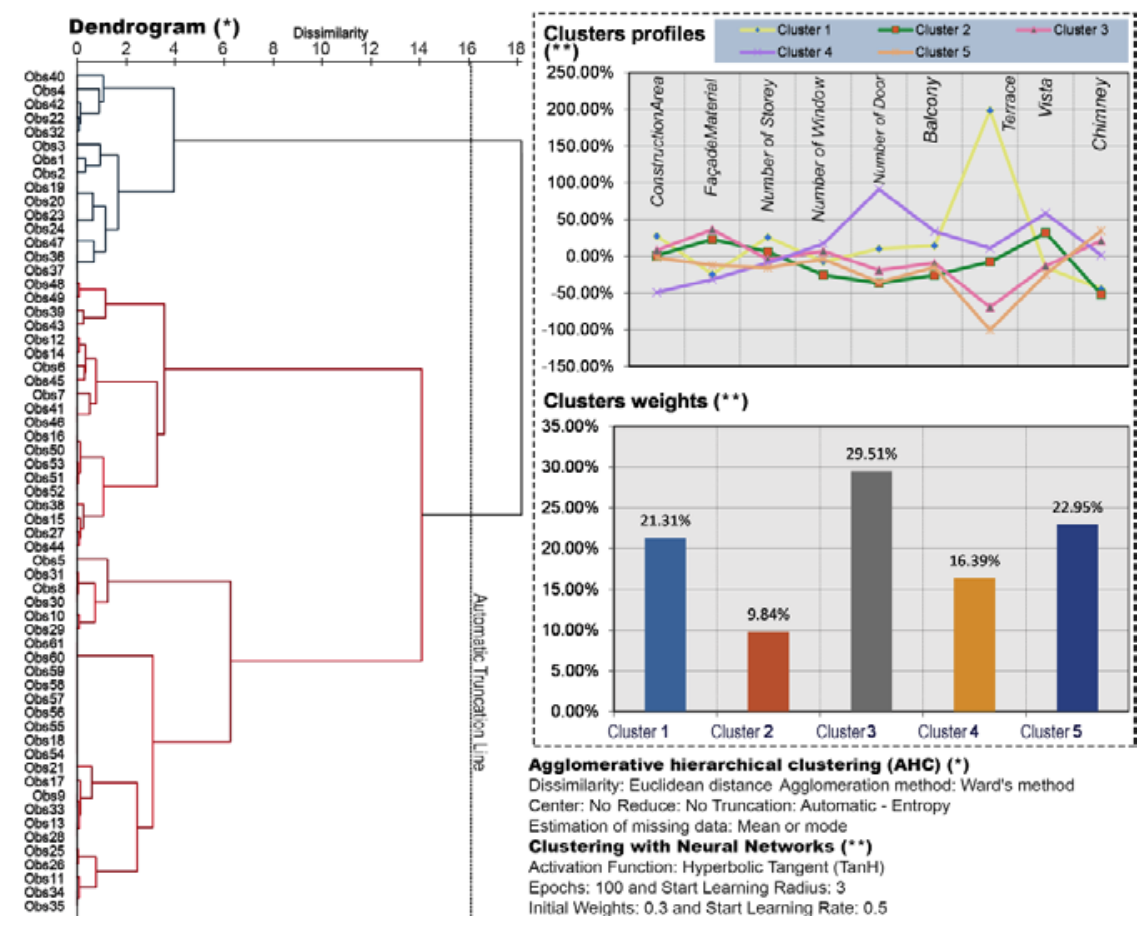

Figure 5. Results of NN-based (right) and Agglomerative Hierarchical clustering Source: Authors 


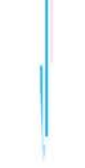

\begin{tabular}{|c|l|c|c|c|c|c|}
\hline Model & Learner & AUC & CA & F1 & Precision & Recall \\
\hline \multirow{2}{*}{$\Delta \begin{array}{l}\text { SqueezeNET } \\
\text { Architecture }\end{array}$} & SVM & 0.855 & 0.792 & 0.791 & 0.797 & 0.792 \\
\cline { 2 - 7 } & Random Forest & 0.727 & 0.640 & 0.640 & 0.641 & 0.640 \\
\cline { 2 - 7 } & Logistic Regression & 0.816 & 0.724 & 0.724 & 0.725 & 0.724 \\
\hline \multirow{3}{*}{$\begin{array}{l}\text { Inception-v3 } \\
\text { Architecture }\end{array}$} & SVM & 0.898 & 0.788 & 0.785 & 0.804 & 0.788 \\
\cline { 2 - 7 } & Random Forest & 0.786 & 0.708 & 0.708 & 0.709 & 0.708 \\
\cline { 2 - 7 } & Logistic Regression & 0.865 & 0.780 & 0.779 & 0.783 & 0.780 \\
\hline
\end{tabular}

\begin{tabular}{|c|c|c|c|c|c|c|c|c|c|}
\hline \multicolumn{6}{|c|}{ Predicted } & \multicolumn{4}{|c|}{ Predicted } \\
\hline$\Delta$ & Contusion Matrix for SVM & Bodrum House & Town House & $\Sigma$ & $\mathrm{Oc}_{\mathrm{c}}$ & onfusion Matrix for SVM & Bodrum House & Town House & $\Sigma$ \\
\hline \multirow[t]{3}{*}{ Actual } & Bodrum House & $83.5 \%$ & $24.1 \%$ & 125 & Actual & Bodrum House & $87.5 \%$ & $26.6 \%$ & 125 \\
\hline & Town House & $16.5 \%$ & $75.9 \%$ & 125 & & Town House & $12.5 \%$ & $73.4 \%$ & 125 \\
\hline & $\Sigma$ & Predicted $^{109}$ & 141 & 250 & & $\Sigma$ & Predicted $^{96}$ & 154 & 250 \\
\hline \multirow{4}{*}{$\underset{\text { Actual }}{\Delta}$} & Confusion Matrix for LR & Bodrum House & Town House & $\Sigma$ & 0 & Confusion Matrix for LR & Bodrum House & Town House & $\Sigma$ \\
\hline & Bodrum House & $73.7 \%$ & $28.8 \%$ & 125 & Actual & Bodrum House & $81.2 \%$ & $24.6 \%$ & 125 \\
\hline & Town House & $26.3 \%$ & $71.2 \%$ & 125 & & Town House & $18.8 \%$ & $75.4 \%$ & 125 \\
\hline & $\Sigma$ & Predicted $^{118}$ & 132 & 250 & & $\Sigma$ & Predicted $^{112}$ & 138 & 250 \\
\hline \multirow{4}{*}{$\underset{\text { Actual }}{\Delta}$} & Confusion Matrix for RF & Bodrum House & Town House & $\Sigma$ & C & Confusion Matrix for $\mathrm{RF}$ & Bodrum House & Town House & $\Sigma$ \\
\hline & Bodrum House & $63.2 \%$ & $35.0 \%$ & 125 & Actual & Bodrum House & $69.4 \%$ & $27.6 \%$ & 125 \\
\hline & Town House & $36.8 \%$ & $65.0 \%$ & 125 & & Town House & $30.6 \%$ & $72.4 \%$ & 125 \\
\hline & $\Sigma$ & 133 & 117 & 250 & & $\Sigma$ & 134 & 116 & 250 \\
\hline
\end{tabular}

Figure 6. Comparative table of different models' scores (upper) and Confusion matrix Source: Authors



Figure 7. Results of the ROC analysis

Source: Authors 
The confusion matrix shows the accuracy of the predicted samples of different learners and models. The highest value of this ratio for Bodrum houses (87.5 \%) was the case where the Inception-v3 network and Support Vector Machine (SVM) algorithm worked together, while the lowest rate (63.2 $\%$ ) was observed as the case where the SqueezeNET network and Random Forest classifier worked together. The area under the ROC Curve (AUC) value should be close to or equal to 1.00 , the maximum AUC value was calculated as 0.898 in this study.

\section{Discussion}

Due to the superior abilities of artificial neural networks in problem-solving skills, both theoretical and implementation areas have been enriched. Artificial intelligence-supported image classification studies and clustering operations are extremely significant for the field of architecture and rural heritage conservation. Besides, they have a facilitating role in the recognition and archiving of a large number of architectural structures and elements in terms of typological studies. In the phases of defining and cataloging architectural constituents, semi-automatic or full-automatic processes can be performed instead of manual with computer-aided/artificial intelligence-aided systems. The findings indicate that the use of computer-aided intelligent systems will contribute positively to the management of the whole process. Within the scope of this study, image classification and clustering procedures were devised by taking into account the architectural façade features of the Bodrum district. In this context, image classification experiments were carried out with deep neural networks on a total of one hundred and twenty-five façade images. Clustering operations were applied to decipher conspicuous patterns from the repository of data derived from the morphological properties of the façades. The construction of a database containing numerical values (textual values converted to numerical values between $[0,1]$ ) for clustering operations contributed positively to the process management. Dissimilarity distributions in the dataset were revealed by agglomerative hierarchical clustering procedures. Moreover, cluster weights and profiles were determined by NNbased clustering operations. Meanwhile, the modified SqueezeNET model and Inception-v3 model were used together with Support Vector Machine (SVM), Random Forest (RF), and Logistic Regression (LR) classifiers. Consequently, relatively successful results were obtained. With the classification processes, it will be possible to identify and categorize Bodrum houses and it might allow us to predict its possible elaborations. The data set in this study is less than five hundred as data augmentation methods such as rotation and translation are not used. In future studies, it is planned to use artificial intelligence to identify architectural styles and typologies by enriching the dataset. 


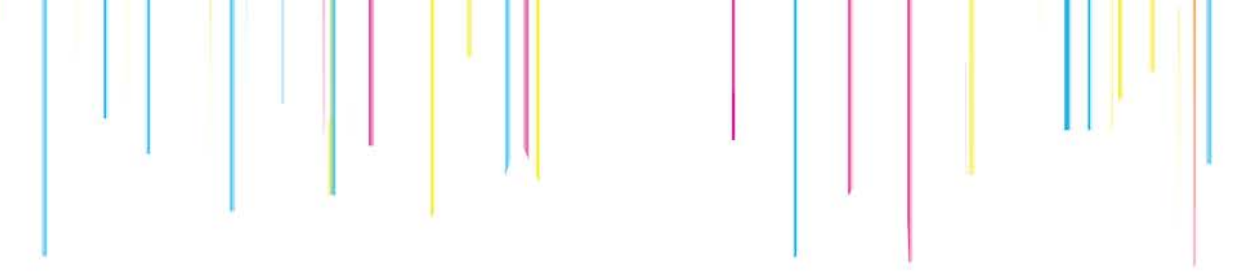

\section{References}

Akçura, N., ve Akçura, T. (1971). Kasaba Ölçeğinde Çevre Değerlerini Koruma Amaçlı Bir İnceleme: Bodrum, Mimarlık Dergisi, 8, s.65-72.

Bektaş, C. (1996). Halk Yapı Sanatından Bir Örnek Bodrum Anadolu Evleri Dizisi-I, Bileşim Yayınevi, İstanbul, s.47-68.

Demšar, J., Curk, T., Erjavec, A., Gorup, Č., Hočevar, T., Milutinovič, M., ...\& Zupan, B. (2013). Orange: data mining toolbox in Python. the Journal of machine Learning research, 14(1), 2349-2353.

Elmas, Ç. (2021). Yapay zeka uygulamaları. Ankara, Seçkin | Teknik.

Fyfe, C. (2000). Artificial neural networks and information theory. University of Paisley.

Gero, J. S. (1996). Artificial intelligence in computer-aided design: Progress and prognosis.

GNU Image Manipulation Program. (2019). GIMP (version 2.10.24). https://www.gimp.org/

He, K., Zhang, X., Ren, S., \& Sun, J. (2016).Deep residual learning for image recognition.In Proceedings of the IEEE conference on computer vision and pattern recognition (pp. 770-778).

Huang, G. B. (2003). Learning capability and storage capacity of two-hidden-layer feedforward networks. IEEE transactions on neural networks, 14(2), 274-281.

Huang, Y. C., Wang, S. Y., Liong, S. T., Huang, C. E., Hsieh, Y. C., Wang, H. Y., ... \&Gan, Y. S. (2020). Who is the designer? Arc-100 database and benchmark on architecture classification. International Journal of Computational Intelligence Systems, 13(1), 1305-1314.

Iandola, F. N., Han, S., Moskewicz, M. W., Ashraf, K., Dally, W. J., \&Keutzer, K. (2016). SqueezeNet: AlexNet-level accuracy with 50x fewer parameters and $<0.5$ MB model size. arXiv preprint arXiv:1602.07360.

Kraus, D. (2014). Consolidated data analysis and presentation using an open-source add-in for the Microsoft Excel ${ }^{\circ}$ spreadsheet software. Medical Writing, 23(1), 2528.

Krizhevsky, A., Sutskever, I., \& Hinton, G. E. (2012). Imagenet classification with deep convolutional neural networks. Advances in neural information processing systems, 25, 1097-1105.

LeCun, Y., Bottou, L., Bengio, Y., \& Haffner, P. (1998). Gradient-based learning applied to document recognition. Proceedings of the IEEE, 86(11), 2278-2324.

Map Maker: Demo Map. (n.d.).Maps.co. https://maps.co/gis/

Meltser, R. D., Banerji, S., \& Sinha, A. (2017, December). What's that Style? A CNNbased Approach for Classification and Retrieval of Building Images. In 2017 Ninth International Conference on Advances in Pattern Recognition (ICAPR) (pp. 16).IEEE. 


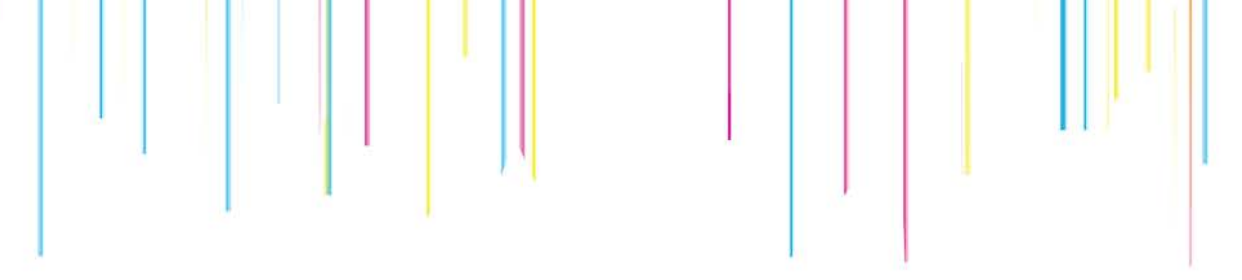

Microsoft Office (2019). (n.d.). Microsoft. https://www.microsoft.com/en-us/microsoft365/get-started-with-office-2019

Mitchell, T. M. (1997). Does machine learning really work?. Al magazine, 18(3), 11-11.

NeuroXL. (2016). Olsoft LLC(version 4.0.6 ). http://www.neuroxl.com/

Nwankpa, C., ljomah, W., Gachagan, A., \& Marshall, S. (2018). Activation functions: Comparison of trends in practice and research for deep learning. arXiv preprint arXiv:1811.03378.

Obeso, A. M., Benois-Pineau, J., Acosta, A. Á. R., \& Vázquez, M. S. G. (2016).Architectural style classification of Mexican historical buildings using deep convolutional neural networks and sparse features. Journal of Electronic Imaging, 26(1), 011016.

PerspectivelmageCorrection.(2021). SourceForge (version 2.0.0.8).[Computer software]. https://sourceforge.net/projects/perspectiveimg/

Simonyan, K., \& Zisserman, A. (2014).Very deep convolutional networks for largescale image recognition. arXiv preprint arXiv:1409.1556.

Szegedy, C., Vanhoucke, V., loffe, S., Shlens, J., \& Wojna, Z. (2016). Rethinking the inception architecture for computer vision.In Proceedings of the IEEE conference on computer vision and pattern recognition (pp. 2818-2826).

Tangram Heightmapper.(n.d.).Github.io. https://tangrams.github.io/heightmapper/Tangram

Tayfur, G. (2014). Soft computing in water resources engineering: Artificial neural networks, fuzzy logic and genetic algorithms. WIT Press.

Wei, F., Li, Y., \& Shamir, L. (2018).Computer Analysis of Architecture Using Automatic Image Understanding. arXiv preprint arXiv:1807.04892.

Wikipedia Contributors. (2019, July 25). Normalization (statistics). Wikipedia; Wikimedia Foundation. https://en.wikipedia.org/wiki/Normalization_(statistics)

Xia, B., Li, X., Shi, H., Chen, S., \& Chen, J. (2020). Style classification and prediction of residential buildings based on machine learning. Journal of Asian Architecture and Building Engineering, 19(6), 714-730.

XIstat | Statistical Software for Excel. (2019, September 29). XIstat (Trial Version), Your Data Analysis Solution. https://www.xlstat.com/en/

Yi, Y. K., Zhang, Y., \& Myung, J. (2020). House style recognition using deep convolutional neural network. Automation in Construction, 118, 103307. 